\title{
Polariton-assisted cooperativity of molecules in microcavities monitored by two-dimensional infrared spectroscopy: Supplementary Information
}

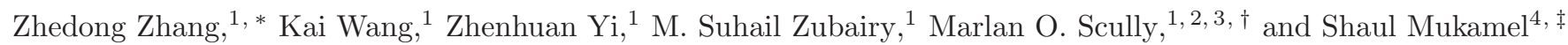 \\ ${ }^{1}$ Institute for Quantum Science and Engineering, \\ Texas A\&M University, College Station, TX 77843, USA \\ ${ }^{2}$ Quantum Optics Laboratory, Baylor Research and Innovation Collaborative, Waco, TX 76704, USA \\ ${ }^{3}$ Department of Mechanical and Aerospace Engineering, \\ Princeton University, Princeton, NJ 08544, USA \\ ${ }^{4}$ Department of Chemistry, Department of Physics and Astronomy, \\ University of California Irvine, Irvine, CA 92697, USA
}

\begin{abstract}
This supplementary information will provide the details about the stochastic models for the hybrid vibration/photon systems of molecules. The derivations for some equations and also some detail analysis of the time- and frequency-resolved spectroscopy for vibrational polaritons are provided in support of the main text.
\end{abstract}

\section{STOCHASTIC MODELS OF VIBRATIONAL POLARITONS}

Dicke model consists of several two-level systems (TLS's) coupled to monochromatic electromagnetic field, which is effective for cavity QED in atoms. When it comes to condensed-phase molecules, the local environment (e.g., solvent and collision) surrounding each individual molecule can drag the vibrational frequency. This results in the frequency fluctuations $\Delta \omega\left(\left\{q_{l}\right\}\right)$ where $q_{l}$ 's are a set of stochastic variables like solvent coordinates accounting for the motion of environment. We will concentrate on the infrared regime hereafter and the model which will be developed can be straightforwardly extended into visible regime including the electronic excitations. With these considerations, the joint molecule/photon system is described by the Hamiltonian

$$
H_{p}=\hbar \sum_{j=1}^{N}\left[\left(\omega_{j}+\delta \omega_{j}(q)-\omega_{c}\right) b_{j}^{\dagger} b_{j}+\Delta_{j} b_{j}^{\dagger} b_{j}^{\dagger} b_{j} b_{j}+g_{j}\left(b_{j}^{\dagger} a+b_{j} a^{\dagger}\right)\right]
$$

in rotating frame of photons+solvent, where $b_{j}$ and $a$ are the bosonic annihilation operators for the vibration of the $j$-th molecule and cavity photons, respectively. $\omega_{j}$ and $\Delta_{j}$ stand for the frequency and anharmonicity of the vibrational mode. $\omega_{c}$ is the photon frequency. The stochastic variables $q_{l}$ s satisfy simple Markovian dynamical rules: the probability of the random variables $q_{l}$ s obeys the master equation

$$
\dot{P}\left(\left\{q_{l}\right\}\right)=\hat{\mathcal{W}} P\left(\left\{q_{l}\right\}\right)
$$

where $\hat{\mathcal{W}}$ is a linear operator. The dynamics is then fully described by the quantum stochastic Liouville equation (QSLE), which was originally proposed for interpreting the spectral line-shape [1,2]. The density matrix at a given time may be written into the joint molecule+bath space. This density matrix has three indices: two represent the ket and bra states of the molecules, while the third denotes the stochastic variables. In the joint space, the total Hamiltonian is time-independent and the dynamics is governed by the QSLE

$$
\frac{\mathrm{d} \rho}{\mathrm{d} t}=\frac{i}{\hbar}\left[\rho, H_{p}\right]+\hat{\mathcal{W}} \rho .
$$

The system density matrix is eventually obtained by the standard prescription of statistical mechanics: average over the initial and sum-over the final states of the stochastic variables.

\section{A. Multi-state random jump model for chemical exchange}

Two types of stochastic models are commonly used in theories of spectral line-shape and diffusion. The first one is the multiple-state jump model, which assumes that the bath hops between $M$ discrete states. This model has

\footnotetext{
* zhedong.zhang@tamu.edu

$\dagger$ scully@tamu.edu

‡smukamel@uci.edu
} 
been applied to describe the chemical exchange processes, causing time-dependent spectral jumps between stable configurations of molecular complexes $[1,3,4]$. The stochastic variables $q_{l}$ s are allowed to take $M_{j}$ discrete values, i.e., $q_{l}=0,1,2, \cdots, M_{j}-1$. Thus the Hamiltonian in Eq.(4) is recast into

$$
H_{p}=\hbar \sum_{j=1}^{N}\left[\left(\omega_{j}+\sum_{l=1}^{M_{j}} \delta \omega_{j}^{(l)} \sigma_{j}^{l l}-\omega_{c}\right) b_{j}^{\dagger} b_{j}+\Delta_{j} b_{j}^{\dagger} b_{j}^{\dagger} b_{j} b_{j}+g_{j}\left(b_{j}^{\dagger} a+b_{j} a^{\dagger}\right)\right]
$$

where $\delta \omega_{j}^{(l)}$ s describe the excitation frequency modulations at the $j$-th molecule and $\sigma_{j}^{l l}$ is the diagonal matrix

$$
\sigma_{j}^{l l}=\left(\begin{array}{ccccc}
0 & & & & \\
& \ddots & & & \\
& & 1 & & \\
& & & \ddots & \\
& & & & 0
\end{array}\right) .
$$

The master equation for bath is

$$
\dot{P}_{n}^{(j)}=\sum_{m=1}^{M} \hat{\mathcal{W}}_{n m}^{(j)} P_{m}^{(j)}
$$

and the QSLE reads

$$
\begin{aligned}
\frac{\mathrm{d} \rho}{\mathrm{d} t}=\frac{i}{\hbar} & {\left[\rho, H_{p}\right]+\sum_{j=1}^{N} \sum_{m>n}\left[\gamma_{m n}^{(j),+}\left(v_{m n}^{(j),+} \rho v_{n m}^{(j),-}-v_{n m}^{(j),-} v_{m n}^{(j),+} \rho\right)\right.} \\
& \left.+\gamma_{n m}^{(j),-}\left(v_{n m}^{(j),-} \rho v_{m n}^{(j),+}-v_{m n}^{(j),+} v_{n m}^{(j),-} \rho\right)\right]+\frac{\omega_{c}}{2 Q}\left(a \rho a^{\dagger}-a^{\dagger} a \rho\right)+\text { h.c. }
\end{aligned}
$$

where $v_{m n}^{(j),+}$ and $v_{n m}^{(j),-}$ denote the raising and lowering operators on $M$ discrete states associated with $q_{j}$ in bath space. $\gamma_{m n}^{(j),+}$ and $\gamma_{n m}^{(j),-}$ are the transition rates for the processes $n \rightarrow m$ and $m \rightarrow n$, respectively. For the finite temperature $T$, the transition rates obey the Boltzmann law

$$
\frac{\gamma_{m n}^{(j),+}}{\gamma_{n m}^{(j),-}}=e^{-\left(\varepsilon_{m}-\varepsilon_{n}\right) / k_{B} T}
$$

and $k_{B}$ is Boltzmann constant. For two-state random jump, Eq.(7) reduces to

$$
\frac{\mathrm{d} \rho}{\mathrm{d} t}=\frac{i}{\hbar}\left[\rho, H_{p}\right]+\sum_{j=1}^{N}\left[\gamma_{j}^{+}\left(\sigma_{j}^{+} \rho \sigma_{j}^{-}-\sigma_{j}^{-} \sigma_{j}^{+} \rho\right)+\gamma_{j}^{-}\left(\sigma_{j}^{-} \rho \sigma_{j}^{+}-\sigma_{j}^{+} \sigma_{j}^{-} \rho\right)\right]+\frac{\omega_{c}}{2 Q}\left(a \rho a^{\dagger}-a^{\dagger} a \rho\right)+\text { h.c. }
$$

which gives the QSLE in the main text. $\gamma_{j}^{+} / \gamma_{j}^{-}=e^{-\varepsilon_{j} / k_{B} T}$ and $\sigma_{j}^{ \pm}$are the Pauli matrices operating in bath space

$$
\sigma_{j}^{+}\left|l_{1}, l_{2}, \cdots, l_{N}\right\rangle=\sqrt{1-l_{j}}\left|l_{1}, l_{2}, \cdots, 1-l_{j}, \cdots, l_{N}\right\rangle ; \quad \sigma_{j}^{-}\left|l_{1}, l_{2}, \cdots, l_{N}\right\rangle=\sqrt{l_{j}}\left|l_{1}, l_{2}, \cdots, 1-l_{j}, \cdots, l_{N}\right\rangle
$$

where $l_{1}, l_{2}, \cdots, l_{N}$ denotes the configurations of the solvent surrounding the individual molecule, and $l_{j}=0,1 ; j=$ $1,2, \cdots, N$. As $\left[H_{p}, \sigma_{j}^{z}\right]=0$, the Hamiltonian $H_{p}$ is block diagonal

$$
H_{p}=\oplus \sum_{\left\{l_{1}, l_{2}, \cdots, l_{N}\right\}} H_{p}^{\left(l_{1}, l_{2}, \cdots, l_{N}\right)}
$$

under the basis $\left|n_{1}, n_{2}, \cdots, n_{N} ; m\right\rangle \otimes\left|l_{1}, l_{2}, \cdots, l_{N}\right\rangle$ where $n_{j}$ and $m$ denote the numbers of vibrational excitations at the $j$-th molecule and photons, respectively. Since the total excitation number $\mathcal{N}=\sum_{j=1}^{N} n_{j}+m$ is conserved, we can consider the ground-state and singly-excited state manifolds only, i.e., $\mathcal{N}=0,1$, for calculating the third-order response function later on. The basis for these manifolds are

$$
\begin{aligned}
& \left|G^{\left(l_{1}, l_{2}, \cdots, l_{N}\right)}\right\rangle=\left|0_{1}, 0_{2}, \cdots, 0_{N} ; 0\right\rangle \otimes\left|l_{1}, l_{2}, \cdots, l_{N}\right\rangle \\
& \left|e_{j}\right\rangle \otimes\left|l_{1}, l_{2}, \cdots, l_{N}\right\rangle=\left\{\left|0_{1}, 0_{2}, \cdots, 1_{j}, \cdots, 0_{N} ; 0\right\rangle \otimes\left|l_{1}, l_{2}, \cdots, l_{N}\right\rangle ;\left|0_{1}, 0_{2}, \cdots, 0_{N} ; 1\right\rangle \otimes\left|l_{1}, l_{2}, \cdots, l_{N}\right\rangle\right\} .
\end{aligned}
$$


Let $\left|\Psi_{k}^{\left(l_{1}, l_{2}, \cdots, l_{N}\right)}\right\rangle$ be the $k$-th eigenstate of $H_{p}^{\left(l_{1}, l_{2}, \cdots, l_{N}\right)}$ in ascending order of the eigenenergies for $\mathcal{N}=1$ manifold. In terms of a linear combination between $\left|e_{j}\right\rangle \otimes\left|l_{1}, l_{2}, \cdots, l_{N}\right\rangle,(j=1,2, \cdots, N+1)$ one has

$$
\left|\Psi_{k}^{\left(l_{1}, l_{2}, \cdots, l_{N}\right)}\right\rangle=\sum_{j=1}^{N+1} C_{j, k}^{\left(l_{1}, l_{2}, \cdots, l_{N}\right)}\left|e_{j}\right\rangle \otimes\left|l_{1}, l_{2}, \cdots, l_{N}\right\rangle
$$

where $C_{j, k}^{\left(l_{1}, l_{2}, \cdots, l_{N}\right)}$ is the unitary matrix diagonalizing $H_{p}^{\left(l_{1}, l_{2}, \cdots, l_{N}\right)}$. Under the resonant condition $\omega_{c}=\omega_{j}$, diagonalization of $H_{p}^{\left(0_{1}, 0_{2}, \cdots, 0_{N}\right)}$ gives rise to two superradiant states termed as lower polariton (LP) and upper polariton (UP). To discuss the dynamics of molecular compounds, Liouville space will be a powerful tool. The time evolution of the joint vibration/photon system is dictated by $|\rho(t)\rangle\rangle=\exp (\hat{L} t)|\rho(0)\rangle\rangle$ where the Liouvillian $\hat{L}$ is given by Eq.(3) in the main text and its elements are

$$
\begin{gathered}
\left\langle\left\langle e_{n^{\prime}} e_{n} ;\left\{l_{1}, l_{2}, \cdots, l_{N}\right\}|\hat{L}| e_{m^{\prime}} e_{m} ;\left\{r_{1}, r_{2}, \cdots, r_{N}\right\}\right\rangle\right\rangle=i \sum_{j=1}^{N}\left(\omega_{j}-\delta \omega_{j}\left(1-2 l_{j}\right)-\omega_{c}\right)\left(\delta_{j n}-\delta_{j n^{\prime}}\right) \delta_{n^{\prime} m^{\prime}} \delta_{n m} \prod_{s=1}^{N} \delta_{l_{s}, r_{s}} \\
\quad+i g_{m} \delta_{n, N+1} \delta_{n^{\prime} m^{\prime}}\left(1-\delta_{m, N+1}\right) \prod_{s=1}^{N} \delta_{l_{s}, r_{s}}+i \sum_{j=1}^{N} g_{j} \delta_{j n} \delta_{n^{\prime} m^{\prime}} \delta_{m, N+1} \prod_{s=1}^{N} \delta_{l_{s}, r_{s}} \\
\quad-i g_{m^{\prime}} \delta_{n^{\prime}, N+1} \delta_{n m}\left(1-\delta_{m^{\prime}, N+1}\right) \prod_{s=1}^{N} \delta_{l_{s}, r_{s}}-i \sum_{j=1}^{N} g_{j} \delta_{j n^{\prime}} \delta_{m^{\prime}, N+1} \delta_{n m} \prod_{s=1}^{N} \delta_{l_{s}, r_{s}} \\
+\delta_{n^{\prime} m^{\prime}} \delta_{n m}\left[\sum_{j=1}^{N} \gamma_{j}\left(\bar{n}_{\nu_{j}}+r_{j}\right) \delta_{1-l_{j}, r_{j}} \prod_{\substack{s=1 \\
(s \neq j)}}^{N} \delta_{l_{s}, r_{s}}-\left(\sum_{j=1}^{N} \gamma_{j}\left(\bar{n}_{\nu_{j}}+l_{j}\right)+\frac{\omega_{c}}{2 Q}\left(\delta_{n^{\prime}, N+1}+\delta_{n, N+1}\right)\right) \prod_{s=1}^{N} \delta_{l_{s}, r_{s}}\right] \\
\left\langle\left\langle G, G ;\left\{l_{1}, l_{2}, \cdots, l_{N}\right\}|\hat{L}| G, G ;\left\{r_{1}, r_{2}, \cdots, r_{N}\right\}\right\rangle\right\rangle=\sum_{j=1}^{N} \gamma_{j}\left(\bar{n}_{\nu_{j}}+r_{j}\right) \delta_{1-l_{j}, r_{j}} \prod_{\substack{s=1 \\
(s \neq j)}}^{N} \delta_{l_{s}, r_{s}}-\sum_{j=1}^{N} \gamma_{j}\left(\bar{n}_{\nu_{j}}+l_{j}\right) \prod_{s=1}^{N} \delta_{l_{s}, r_{s}} \\
\left\langle\left\langle G, G ;\left\{l_{1}, l_{2}, \cdots, l_{N}\right\}|\hat{L}| e_{m^{\prime}} e_{m} ;\left\{r_{1}, r_{2}, \cdots, r_{N}\right\}\right\rangle\right\rangle=\frac{\omega_{c}}{Q} \delta_{m^{\prime}, N+1} \delta_{m, N+1} \prod_{s=1}^{N} \delta_{l_{s}, r_{s}} \\
\left\langle\left\langle e_{n^{\prime}} e_{n} ;\left\{l_{1}, l_{2}, \cdots, l_{N}\right\}|\hat{L}| G, G ;\left\{r_{1}, r_{2}, \cdots, r_{N}\right\}\right\rangle\right\rangle=0
\end{gathered}
$$

and the block for optical coherence

$$
\begin{aligned}
\left\langle\left\langle G, e_{n} ;\left\{l_{1}, l_{2}, \cdots,\right.\right.\right. & \left.\left.\left.l_{N}\right\}|\hat{L}| G, e_{m} ;\left\{r_{1}, r_{2}, \cdots, r_{N}\right\}\right\rangle\right\rangle=i \sum_{j=1}^{N}\left(\omega_{j}-\delta \omega_{j}\left(1-2 l_{j}\right)-\omega_{c}\right) \delta_{j n} \delta_{n m} \prod_{s=1}^{N} \delta_{l_{s}, r_{s}} \\
& +i g_{m} \delta_{n, N+1}\left(1-\delta_{m, N+1}\right) \prod_{s=1}^{N} \delta_{l_{s}, r_{s}}+i \sum_{j=1}^{N} g_{j} \delta_{j n} \delta_{m, N+1} \prod_{s=1}^{N} \delta_{l_{s}, r_{s}} \\
+ & \delta_{n m}\left[\sum_{j=1}^{N} \gamma_{j}\left(\bar{n}_{\nu_{j}}+r_{j}\right) \delta_{1-l_{j}, r_{j}} \prod_{\substack{s=1 \\
s \neq j}}^{N} \delta_{l_{s}, r_{s}}-\left(\sum_{j=1}^{N} \gamma_{j}\left(\bar{n}_{\nu_{j}}+l_{j}\right)+\frac{\omega_{c}}{2 Q} \delta_{n, N+1}\right) \prod_{s=1}^{N} \delta_{l_{s}, r_{s}}\right]
\end{aligned}
$$

where the abbreviations are used: $\left.\left.\left.\left.\left|e_{n^{\prime}} e_{n} ;\left\{l_{1}, l_{2}, \cdots, l_{N}\right\}\right\rangle\right\rangle \equiv\left|e_{n^{\prime}} e_{n}\right\rangle\right\rangle \otimes\left|\left\{l_{1}, l_{2}, \cdots, l_{N}\right\},\left\{l_{1}, l_{2}, \cdots, l_{N}\right\}\right\rangle\right\rangle,\left|G, G ;\left\{l_{1}, l_{2}, \cdots, l_{N}\right\}\right\rangle\right\rangle \equiv$ $\left.|G, G\rangle\rangle \otimes\left|\left\{l_{1}, l_{2}, \cdots, l_{N}\right\},\left\{l_{1}, l_{2}, \cdots, l_{N}\right\}\right\rangle\right\rangle$ and $\left.\left.\left.\left|G, e_{n} ;\left\{l_{1}, l_{2}, \cdots, l_{N}\right\}\right\rangle\right\rangle \equiv\left|G, e_{n}\right\rangle\right\rangle \otimes\left|\left\{l_{1}, l_{2}, \cdots, l_{N}\right\},\left\{l_{1}, l_{2}, \cdots, l_{N}\right\}\right\rangle\right\rangle$. Eq. $(14,15)$ dictate the couplings between polaritons and dark states under the dynamical disorder which erodes the collective nature of the vibrational polaritons. 

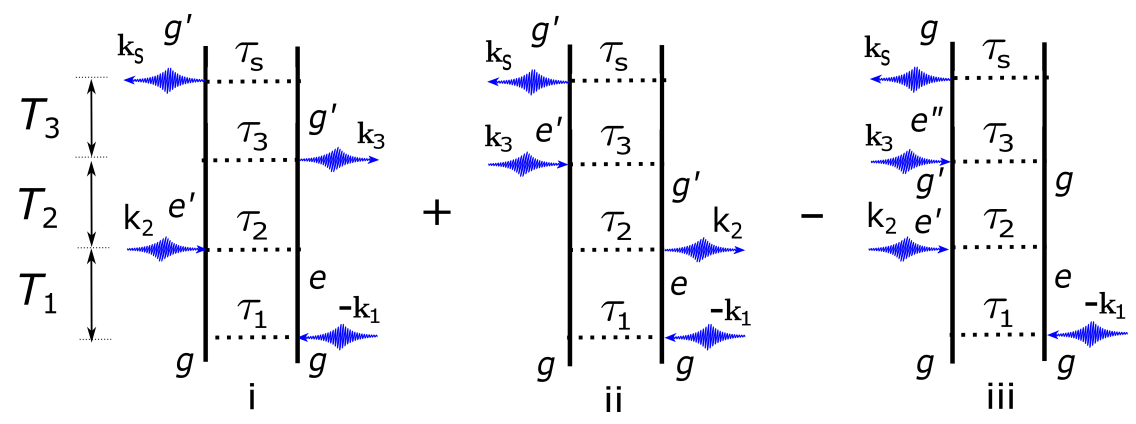

FIG. 1: Double-side Feynman diagrams for photon-echo signal of 2D-IR spectra. Processes (i), (ii) and (iii) correspond to excited-state relaxation (ESR), solvent relaxation (SR) and excited-state decay (ESD), respectively. The (iii) are due to cavity leakage.

\section{B. Spectral diffusion model with continuous stochastic variables}

In the second model, the solvent motion surrounding individual molecule is described by an over-damped Brownian oscillator associated with the dimensionless continuous stochastic variables $Q_{l}, P_{l}$ s. This can describe the spectral diffusion processes. The QSLE was recently applied to model the signatures of conformational fluctuations in trialanine in water, incorporating the Brownian oscillator coordinate model of fluctuations [5]. The full Hamiltonian reads $H=H_{p}+H_{s}$ where

$$
\begin{aligned}
& H_{p}=\hbar \sum_{j=1}^{N}\left[\left(\omega_{j}+\delta \omega_{j} Q_{j}-\omega_{c}\right) b_{j}^{\dagger} b_{j}+\Delta_{j} b_{j}^{\dagger} b_{j}^{\dagger} b_{j} b_{j}+\left(b_{j}^{\dagger} a+b_{j} a^{\dagger}\right)\right] \\
& H_{s}=\hbar \sum_{j=1}^{N} \frac{v_{j}}{2}\left(P_{j}^{2}+Q_{j}^{2}\right)
\end{aligned}
$$

in the rotating frame of photon. Here a linear dependence on stochastic variables was assumed for the frequency fluctuations, i.e., $\Delta \omega_{j}\left(Q_{j}\right) \simeq \delta \omega_{j} Q_{j} . P_{j}, Q_{j}$ are the dimensionless momentum and coordinate operators, respectively. We further assume that the solvent undergoes an Ornstein-Uhlenbeck process and is described the Markovian Langevin equations

$$
\dot{Q}_{j}=v_{j} P_{j}-\gamma_{j} Q_{j}+f_{j}(t) ; \quad \dot{P}_{j}=-v_{j} Q_{j}-\Gamma_{j} P_{j}+F_{j}(t)
$$

where $f_{j}(t)$ and $F_{j}(t)$ represent the white-noise random forces: $\left\langle f_{j}(t) f_{j}\left(t^{\prime}\right)\right\rangle=2 D_{j} \delta\left(t-t^{\prime}\right),\left\langle F_{j}(t) F_{j}\left(t^{\prime}\right)\right\rangle=2 \mathcal{D}_{j} \delta\left(t-t^{\prime}\right)$. For the reduced description of solvent motion, it may be advantageous to use the Wigner phase space representation that is particularly suitable for semiclassical approximations. The Wigner representation of an operator $A$ is obtained by the Fourier transform of the coordinate representation

$$
A(\mathbf{p}, \mathbf{q})=\int_{-\infty}^{\infty} e^{-i \mathbf{p} \cdot \mathbf{s}}\langle\mathbf{q}+\mathbf{s} / 2|A| \mathbf{q}-\mathbf{s} / 2\rangle \mathrm{d} \mathbf{s}
$$

which gives the average of the operator

$$
\langle A\rangle=\iint \rho(\mathbf{p}, \mathbf{q}) A(\mathbf{p}, \mathbf{q}) \mathrm{d} \mathbf{p} \mathrm{d} \mathbf{q} .
$$

Under the classical description of solvent motions, we consider the following model for the vibrational Hamiltonian $H^{(s c)}=H_{p}^{(s c)}+h_{s}$ where

$$
\begin{aligned}
& H_{p}^{(s c)}=\hbar \sum_{j=1}^{N}\left[\left(\omega_{j}+\delta \omega_{j} q_{j}-\omega_{c}\right) b_{j}^{\dagger} b_{j}+\Delta_{j} b_{j}^{\dagger} b_{j}^{\dagger} b_{j} b_{j}+\left(b_{j}^{\dagger} a+b_{j} a^{\dagger}\right)\right] \\
& h_{s}=\hbar \sum_{j=1}^{N} \frac{v_{j}}{2}\left(p_{j}^{2}+q_{j}^{2}\right)
\end{aligned}
$$



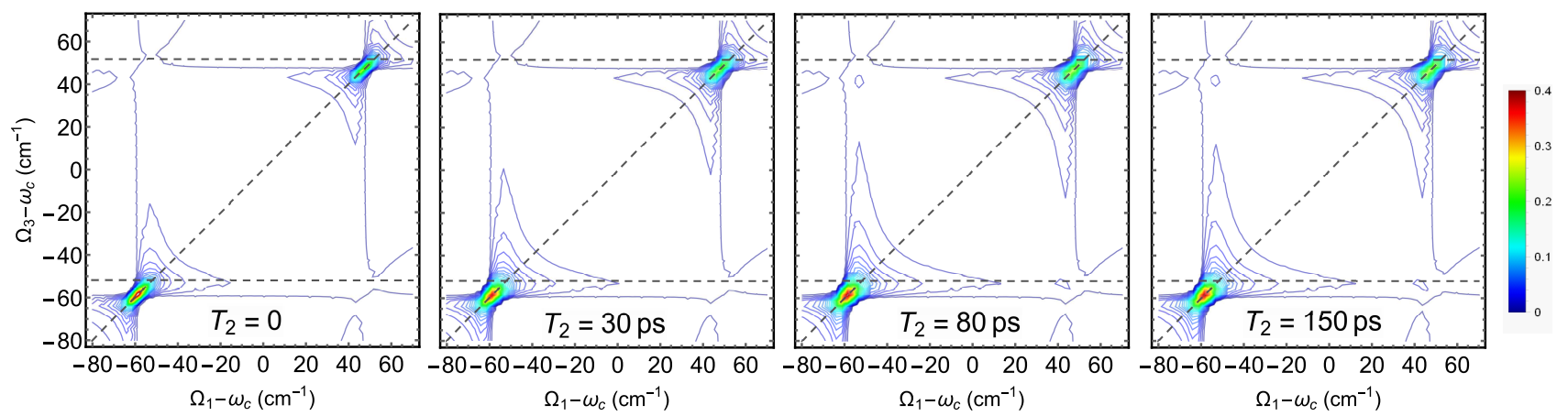

FIG. 2: 2D-IR signal variation with $T_{2}$ delay given by Eq.(5) in main text, for three $\mathrm{W}(\mathrm{CO})_{6}$ molecules placed in an infrared microcavity with a linear fashion along the cavity axis. Coupling of each molecule to cavity is strong than vibrational frequency fluctuation, i.e., $g_{j}>\delta \omega_{j}$. Thermal distribution of solvent prior to pulse action is included under room temperature. $g_{j}=30 \mathrm{~cm}^{-1}$. Other molecule parameters are the same as Fig. 3 in main text; Pulse shape is set to be Gaussian with the paramters $w_{1}=w_{2}=w_{3}=w_{l o}=100 \mathrm{~cm}^{-1}, \omega_{1}=\omega_{2}=1983 \mathrm{~cm}^{-1}$ and $\omega_{3}=\omega_{l o}=1993 \mathrm{~cm}^{-1}$.

Here $p_{j}, q_{j}$ are c-numbers and obey the classical Langevin equation

$$
\dot{q}_{j}=v_{j} p_{j}-\gamma_{j} q_{j}+f_{j}(t) ; \quad \dot{p}_{j}=-v_{j} q_{j}-\Gamma_{j} p_{j}+F_{j}(t) .
$$

The corresponding Fokker-Planck equation for the probability of $p_{j}, q_{j}$ reads

$$
\frac{\partial P_{j}}{\partial t}=\left(\frac{\partial}{\partial p_{j}}\left(\Gamma_{j} p_{j}+v_{j} q_{j}\right)+\frac{\partial}{\partial q_{j}}\left(-v_{j} p_{j}+\gamma_{j} q_{j}\right)+\mathcal{D}_{j} \frac{\partial^{2}}{\partial p_{j}^{2}}+D_{j} \frac{\partial^{2}}{\partial q_{j}^{2}}\right) P_{j} .
$$

and then the QSLE can be found

$$
\begin{aligned}
\frac{\partial}{\partial t} \rho(p, q)= & \frac{i}{\hbar}\left[\rho(p, q), H_{p}^{(s c)}\right]+\frac{\omega_{c}}{2 Q}\left(2 a \rho(p, q) a^{\dagger}-a^{\dagger} a \rho(p, q)-\rho(p, q) a^{\dagger} a\right) \\
& +\sum_{j=1}^{N}\left(\frac{\partial}{\partial p_{j}}\left(\Gamma_{j} p_{j}+v_{j} q_{j}\right)+\frac{\partial}{\partial q_{j}}\left(-v_{j} p_{j}+\gamma_{j} q_{j}\right)+\mathcal{D}_{j} \frac{\partial^{2}}{\partial p_{j}^{2}}+D_{j} \frac{\partial^{2}}{\partial q_{j}^{2}}\right) \rho(p, q)
\end{aligned}
$$

Since the solvent moves much slower than the intramolecular vibrations, we can properly neglect the contribution of solvent kinetic energy, namely, $p_{j} \simeq 0$ and $\rho(p, q) \simeq \rho(q)$. Thereby the QSLE for intramolecular vibrations reads

$$
\frac{\partial}{\partial t} \rho(q)=\frac{i}{\hbar}\left[\rho(q), H_{p}^{(s c)}\right]+\frac{\omega_{c}}{2 Q}\left(2 a \rho(q) a^{\dagger}-a^{\dagger} a \rho(q)-\rho(q) a^{\dagger} a\right)+\sum_{j=1}^{N}\left(\gamma_{j} \frac{\partial}{\partial q_{j}} q_{j}+D_{j} \frac{\partial^{2}}{\partial q_{j}^{2}}\right) \rho(q)
$$

which is hard to solve, except the case $g_{j}=0, \Delta_{j}=0$. The numerical methods for solving Eq.(24) refer to Ref.[6], where the computation is heavily cost.

\section{THE THIRD-ORDER RESONANT INFRARED SPECTROSCOPY FOR VIBRATIONAL POLARITONS}

To gain more information regarding the relaxation of vibrational polaritons beyond the scope of TFRPS, i.e., line broadening and transfer pathways, we will essentially develop a 2D-IR spectroscopy for the joint vibration/photon system by incorporating the disorder effect. The three related processes of excited-state relaxation (ESR), solvent relaxation (SR) and excited-state decay (ESD) are displayed in Fig.1. The sample interacts with three time-ordered pulses by means of dipolar coupling

$$
U_{\text {int }}(t)=\sum_{j=1}^{3} \mu^{(j),+}(t) \mathcal{E}\left(t-\tau_{j}\right) e^{i\left(\mathbf{k}_{j} \cdot \mathbf{r}-\omega_{j}\left(t-\tau_{j}\right)\right)}+\text { h.c. }
$$


where $\mu^{(j),+}(t)=\boldsymbol{\mu}^{+}(t) \cdot \mathbf{e}_{j}$ and $\mathbf{e}_{j}$ is the unit polarization vector of the $j$-th pulse. The photon echoes field interferes in phase with the fourth pulse (local oscillator), and the signal is given by

$$
\begin{aligned}
S_{I}\left(T_{3}, T_{2}, T_{1}\right)= & \int \mathrm{d}^{3} \mathbf{r} \int_{-\infty}^{\infty} \mathrm{d} t\left\langle\mu^{(l o),-}\right\rangle_{\rho(t)} E_{l o}^{*}(t-\tau) e^{-i \mathbf{k}_{l o} \cdot \mathbf{r}} \\
= & \frac{8 \pi^{3}}{\hbar^{3}} \delta\left(\mathbf{k}_{l o}-\mathbf{k}_{3}-\mathbf{k}_{2}+\mathbf{k}_{1}\right) \int_{-\infty}^{\infty} \mathrm{d} t \int_{0}^{\infty} \mathrm{d} t_{3} \int_{0}^{\infty} \mathrm{d} t_{2} \int_{0}^{\infty} \mathrm{d} t_{1} R^{(3)}\left(t_{3}, t_{2}, t_{1}\right) \\
& \quad \times E_{l o}^{*}(t-\tau) E_{3}\left(t-t_{3}-\tau_{3}\right) E_{2}\left(t-t_{3}-t_{2}-\tau_{2}\right) E_{1}^{*}\left(t-t_{3}-t_{2}-t_{1}-\tau_{1}\right)
\end{aligned}
$$

and the dynamical information of molecules is contained in the third-order response function $R^{(3)}\left(t_{3}, t_{2}, t_{1}\right)=$ $R_{1}\left(t_{3}, t_{2}, t_{1}\right)+R_{2}\left(t_{3}, t_{2}, t_{1}\right)-R_{3}\left(t_{3}, t_{2}, t_{1}\right)$ where

$$
\begin{aligned}
& R_{1}\left(t_{3}, t_{2}, t_{1}\right)=\left\langle\left\langle 1\left|\mu_{L}^{(l o),-} \mathcal{G}\left(t_{3}\right) \mu_{R}^{(3),+} \mathcal{G}\left(t_{2}\right) \mu_{L}^{(2),+} \mathcal{G}\left(t_{1}\right) \mu_{R}^{(1),-}\right| G, G\right\rangle\right\rangle \\
& R_{2}\left(t_{3}, t_{2}, t_{1}\right)=\left\langle\left\langle 1\left|\mu_{L}^{(l o),-} \mathcal{G}\left(t_{3}\right) \mu_{L}^{(3),+} \mathcal{G}\left(t_{2}\right) \mu_{R}^{(2),+} \mathcal{G}\left(t_{1}\right) \mu_{R}^{(1),-}\right| G, G\right\rangle\right\rangle \\
& R_{3}\left(t_{3}, t_{2}, t_{1}\right)=\left\langle\left\langle 1\left|\mu_{L}^{(l o),-} \mathcal{G}\left(t_{3}\right) \mu_{L}^{(3),+} \mathcal{G}\left(t_{2}\right) \mu_{L}^{(2),+} \mathcal{G}\left(t_{1}\right) \mu_{R}^{(1),-}\right| G, G\right\rangle\right\rangle .
\end{aligned}
$$

The $R_{1}, R_{2}$ and $R_{3}$ correspond to the ESR, SR and ESD, respectively. $\left.\left.|G, G\rangle\right\rangle=\sum_{J} P_{J}\left|G^{(J)}, G^{(J)}\right\rangle\right\rangle$. $P_{J}$ denotes the statistical probability of system at $\left.\left|G^{(J)}, G^{(J)}\right\rangle\right\rangle$ prior to the pulse actions, and is given by Eq.(6) in the main text for thermal equilibrium. In our model the $R_{2}$ contains the information about the solvent relaxation itself, nothing to do with the vibrational polaritons. This contributes as a background which can be deduced from the full signal by running a control simulation. Under the impulsive approximation we insert the dynamics $\left.|\rho(t)\rangle\rangle=e^{\hat{L} t}|\rho(0)\rangle\right\rangle$ with Eq. $(14,15)$ into $R^{(3)}\left(t_{3}, t_{2}, t_{1}\right)$ and carry out the multifold convolution with respect to pulse envelopes. Some algebra leads to the $2 \mathrm{D}$ signal

$$
\begin{aligned}
S_{I}\left(\Omega_{3}, T_{2}, \Omega_{1}\right)= & -2 \operatorname{Im} \int_{0}^{\infty} \mathrm{d} T_{3} \int_{0}^{\infty} \mathrm{d} T_{1} S_{I}\left(T_{3}, T_{2}, T_{1}\right) e^{i\left(\Omega_{3} T_{3}+\Omega_{1} T_{1}\right)} \\
= & \frac{16 \pi^{3}}{\hbar^{3}} \delta\left(\mathbf{k}_{l o}-\mathbf{k}_{3}-\mathbf{k}_{2}+\mathbf{k}_{1}\right) \operatorname{Re}\left[\left(\sum_{r} \sum_{i, i^{\prime}=1}^{N+1} \sum_{J} \sum_{j, j^{\prime}=1}^{N+1} \frac{V_{(l o, i}^{(r), *} V_{(3), i^{\prime}}^{(r)} V_{(2), j^{\prime}}^{(J)} V_{(1), j}^{(J), *}}{\left(\Omega_{3}-\omega_{i}^{(r)}+i \gamma_{i}^{(r)}\right)\left(\Omega_{1}+\omega_{j}^{(J)}+i \gamma_{j}^{(J)}\right)} P_{J}\right.\right. \\
& \times\left\langle\left\langle\psi_{i}^{(r)}, \psi_{i^{\prime}}^{(r)}\left|\mathcal{G}\left(T_{2}\right)\right| \psi_{j^{\prime}}^{(J)}, \psi_{j}^{(J)}\right\rangle\right\rangle+\sum_{r} \sum_{i=1}^{N+1} \sum_{J} \sum_{j=1}^{N+1} \frac{V_{(l o), i}^{(r), *} V_{(3), i}^{(r)} V_{(2), j}^{(J)} V_{(1), j}^{(J), *}}{\left(\Omega_{3}-\omega_{i}^{(r)}+i \gamma_{i}^{(r)}\right)\left(\Omega_{1}+\omega_{j}^{(J)}+i \gamma_{j}^{(J)}\right)} P_{J}^{N} \\
& \times \prod_{s=1}^{N}\left\langle\left\langle r_{s}, r_{s}\left|\mathcal{G}_{s}^{(g g)}\left(T_{2}\right)\right| J_{s}, J_{s}\right\rangle\right\rangle-\sum_{r} \sum_{i=1}^{N+1} \sum_{J}^{N+1} \frac{V_{\left(l o, j^{\prime}=1\right.}^{(r), *} V_{(3), i}^{(r)} V_{(2), j^{\prime}}^{(J)} V_{(1), j}^{(J), *}}{\frac{\left.\Omega_{3}-\omega_{i}^{(r)}+i \gamma_{i}^{(r)}\right)\left(\Omega_{1}+\omega_{j}^{(J)}+i \gamma_{j}^{(J)}\right)}{P} P_{J}} \\
& \left.\left.\times\left\langle\left\langle G^{(r)}, G^{(r)}\left|\mathcal{G}\left(T_{2}\right)\right| \psi_{j^{\prime}}^{(J)}, \psi_{j}^{(J)}\right\rangle\right\rangle\right) \tilde{\mathcal{E}}_{l o}^{*}\left(\omega_{i}^{(r)}-\omega_{l o}\right) \tilde{\mathcal{E}}_{3}\left(\omega_{i}^{(r)}-\omega_{3}\right) \tilde{\mathcal{E}}_{2}\left(\omega_{j}^{(J)}-\omega_{2}\right) \tilde{\mathcal{E}}_{1}^{*}\left(\omega_{j}^{(J)}-\omega_{1}\right)\right]
\end{aligned}
$$

by Fourier transform with respect to $T_{1}$ and $T_{3}$ delays, where the Green's function during $T_{2}$ delay reads

$$
\begin{aligned}
&\left\langle\left\langle\psi_{i}^{(r)}, \psi_{i^{\prime}}^{(r)}|\mathcal{G}(t)| \psi_{j}^{(J)}, \psi_{j^{\prime}}^{(J)}\right\rangle\right\rangle= \sum_{m, n=1}^{N+1} \sum_{k, l=1}^{N+1} \sum_{u=1}^{\operatorname{dim}(L)} S_{\left\langle e_{m} e_{n}, r\right\rangle ; u} e^{\nu_{u} t} S_{u ;\left\langle e_{k} e_{l}, J\right\rangle}^{-1} C_{e_{k}, j}^{(J), *} C_{e_{l}, j^{\prime}}^{(J)} C_{e_{m}, i}^{(r)} C_{e_{n}, i^{\prime}}^{(r), *} \\
&\left\langle\left\langle G^{(r)}, G^{(r)}|\mathcal{G}(t)| \psi_{j^{\prime}}^{(J)}, \psi_{j}^{(J)}\right\rangle\right\rangle= \frac{\omega_{c}}{Q} \sum_{q, P} \sum_{k, l=1}^{N+1} \sum_{u=1}^{\operatorname{dim}(L)} \prod_{s=1}^{N}\left\langle\left\langle r_{s}, r_{s}\left|\mathcal{G}_{s}^{(g g)}(t)\right| q_{s}, q_{s}\right\rangle\right\rangle S_{\left\langle e_{N+1} e_{N+1}, P\right\rangle ; u} S_{u ;\left\langle e_{k} e_{l}, J\right\rangle}^{-1} \\
& \times\left(\int_{0}^{t} \mathrm{~d} t^{\prime} e^{\nu_{u} t^{\prime}} \prod_{w=1}^{N}\left\langle\left\langle q_{w}, q_{w}\left|\mathcal{G}_{w}^{(q q)}\left(-t^{\prime}\right)\right| P_{w}, P_{w}\right\rangle\right\rangle\right) C_{e_{k}, j^{\prime}}^{(J), *} C_{e_{l}, j}^{(J)} \\
& \mathcal{G}_{s}^{(g g)}(t)=\frac{1}{2 \bar{n}_{v_{s}}+1}\left(\begin{array}{cc}
\bar{n}_{v_{s}}+1 \\
\bar{n}_{v_{s}} \\
\bar{n}_{v_{s}}+1 \\
\bar{n}_{v_{s}}
\end{array}\right)+\frac{e^{-\gamma_{s}\left(2 \bar{n}_{v_{s}}+1\right) t}}{2 \bar{n}_{v_{s}}+1}\left(\begin{array}{c}
\bar{n}_{v_{s}}-\bar{n}_{v_{s}}-1 \\
-\bar{n}_{v_{s}} \\
\bar{n}_{v_{s}}+1
\end{array}\right)
\end{aligned}
$$



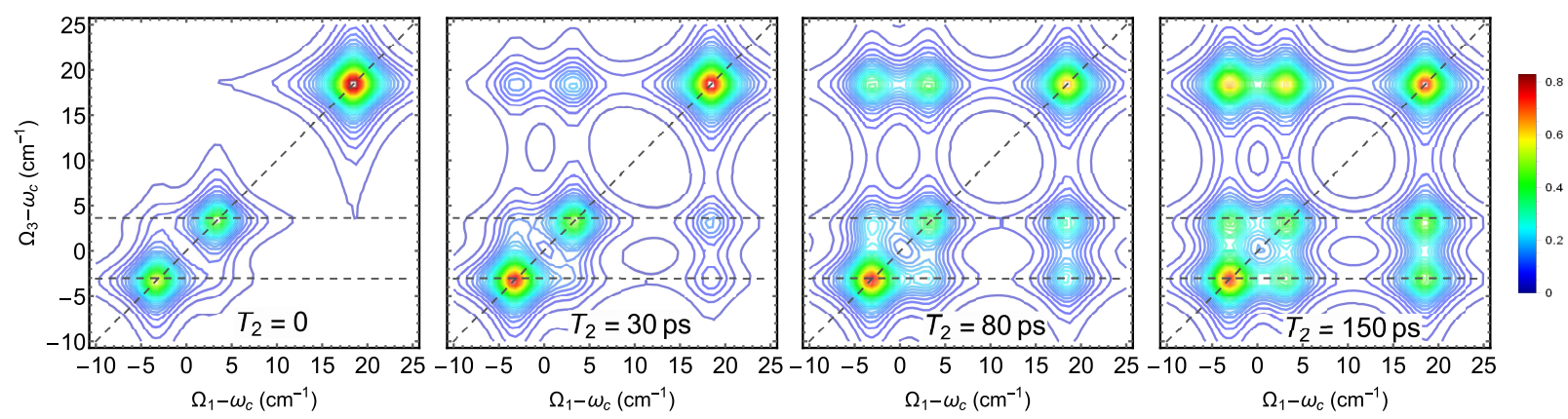

FIG. 3: 2D pump-probe signal variation with $T_{2}$ delay, for three $\mathrm{W}(\mathrm{CO})_{6}$ molecules placed in an infrared microcavity with a linear fashion along the cavity axis. Thermal distribution of solvent prior to pulse action is included under room temperature. $\Omega_{1}$ and $\Omega_{3}$ correspond to excitation and emission frequencies, respectively. Molecule parameters are the same as Fig.3 in main text; Pulse shape is set to be Gaussian with the paramters $w_{1}=w_{2}=w_{3}=w_{l o}=50 \mathrm{~cm}^{-1}, \omega_{1}=\omega_{2}=1983 \mathrm{~cm}^{-1}$ and $\omega_{3}=\omega_{l o}=1993 \mathrm{~cm}^{-1}$.

and $\nu_{n}$ 's are the eigenvalues of Liouvillian $\hat{L}$ in Eq.(14), which governs the dynamics of the joint vibration/photon system during $T_{2}$ delay.

\section{STRONG COUPLING BETWEEN SINGLE MOLECULE AND CAVITY MODE}

To further illustrate the polariton-assited cooperative nature against solvent-induced disorder, we plot in Fig.3 the tomographies of $2 \mathrm{D}$-IR signal $\operatorname{Im}\left[S_{I}\left(\Omega_{3}, T_{2}, \Omega_{1}\right)\right]$ for various $T_{2}$ within the different parameter regime from Fig.2 in main text, whereas the coupling between each molecule to cavity is stronger than the vibrational frequency fluctuation, i.e., $g_{j}>\delta \omega_{j}$. The results prominently resolve the two polariton peaks separated by $\simeq 2 g \sqrt{N}=104 \mathrm{~cm}^{-1}$ and the "dark modes" information are smeared out. This is to say that the strong coupling of single molecule to cavity modes, i.e. $g_{j}>\delta \omega_{j}$, can enhance the cooperativity between molecules.

\section{PUMP-PROBE SPECTRA FOR VIBRATIONAL POLARITONS}

The 2D spectroscopy reduces to the pump-probe scheme when the first two pulses are produced from one single laser beam and are incident in parallel, i.e., $\mathbf{k}_{1}=\mathbf{k}_{2}$. This has been indicated in recent experiments. Here we plot in Fig.3 the pump-probe signal given the phase matching $\mathbf{k}_{s}=\mathbf{k}_{3}$, which may be achieved by combining $S_{I}$ in Eq.(28) with the $S_{I I}$ signal with phase matching $\mathbf{k}_{I I}=\mathbf{k}_{1}-\mathbf{k}_{2}+\mathbf{k}_{3}$, given $\mathbf{k}_{1}=\mathbf{k}_{2}$. From Fig.3 one cannot resolve the homogeneous and inhomogeneous mechanisms of line-broadening. Apart from this, all other dynamical information of the system of interest are the same as that given by Fig.3 in main text.

[1] R. Kubo, J. Math. Phys. 4, 174-183 (1963)

[2] Y. Tanimura, J. Phys. Soc. Jpn. 75, 082001-082039 (2006)

[3] P. W. Anderson, B. I. Halperin and C. M. Varma, Philos. Mag. 25, 1-9 (1971)

[4] F. Sanda and S. Mukamel, J. Chem. Phys. 125, 014507-014518 (2006)

[5] T. L. C. Jansen, W. Zhuang and S. Mukamel, J. Chem. Phys. 121, 10577-10598 (2004)

[6] D. Abramavicius, B. Palmieri, D. V. Voronine, F. Sanda and S. Mukamel, Chem. Rev. 109, 2350-2408 (2009)

[7] S. Mukamel, Principles of Nonlinear Optical Spectroscopy (Oxford University Press, New York, 1995)

[8] Y. J. Yan and S. Mukamel, J. Chem. Phys. 94, 179-190 (1991) 